\title{
Anti-nociceptive effect of dexmedetomidine in a rat model of monoarthritis via suppression of the TLR4/NF-kB p65 pathway
}

\author{
DONG JI $^{1 *}$, YALAN ZHOU ${ }^{1 *}$, SHUANGSHUANG LI ${ }^{1}$, DAI LI ${ }^{1}$, HUI CHEN $^{1}$, \\ YUANCHANG XIONG ${ }^{1}$, YUQIU ZHANG ${ }^{2}$ and HUA XU ${ }^{1}$ \\ ${ }^{1}$ Department of Anesthesiology, Changhai Hospital, Second Military Medical University, Shanghai 200433; \\ ${ }^{2}$ Institute of Neurobiology, Institutes of Brain Science and State Key Laboratory of \\ Medical Neurobiology, Fudan University, Shanghai 200032, P.R. China
}

Received October 17, 2016; Accepted June 2, 2017

DOI: $10.3892 / e t m .2017 .5196$

\begin{abstract}
As a therapeutic target for neuropathic pain, the anti-nociceptive effects of $\alpha 2$-adrenoceptors ( $\alpha 2 \mathrm{AR})$ have attracted attention. Dexmedetomidine (DEX), a potent and highly selective $\alpha 2 \mathrm{AR}$ agonist, has exhibited significant analgesic effects in neuropathic pain, but the underlying mechanism has remained elusive. The present study investigated the effect of DEX on Toll-like receptor (TLR)4 and nuclear factor (NF)- $\kappa$ B p65 expression, as well as the production of pro-inflammatory cytokines. The rat monoarthritis (MA) model was induced by intra-articular injection of complete Freund's adjuvant (CFA) at the ankle joint. After induction of MA, the rats were intrathecally treated with normal saline or DEX $(2.5 \mu \mathrm{g})$ for 3 consecutive days. The concentration of interleukin- $1 \beta$ and -6 as well as tumor necrosis factor- $\alpha$ was examined by ELISA. The expression levels of TLR4 and NF- $\kappa \mathrm{B}$ p 65 were determined by western blot analysis and immunohistochemistry. The results indicated that the pro-inflammatory cytokines TLR4 and NF- $\mathrm{B}$ p65 were significantly upregulated in MA rats. DEX treatment markedly reduced mechanical and thermal hyperalgesia, suppressed MA-induced elevation of the pro-inflammatory cytokines and inhibited the TLR4/NF- $\kappa$ B p65 pathway, while these effects were blocked by pre-treatment with the selective $\alpha 2 \mathrm{AR}$ antagonist BRL44408 $(15 \mu \mathrm{g})$ at $30 \mathrm{~min}$ prior to CFA
\end{abstract}

Correspondence to: Dr Hua $\mathrm{Xu}$, Department of Anesthesiology, Changhai Hospital, Second Military Medical University, 168 Changhai Road, Shanghai 200433, P.R. China

E-mail: pshhuaxu@163.com

Dr Yuqiu Zhang, Institute of Neurobiology, Institutes of Brain Science and State Key Laboratory of Medical Neurobiology, Fudan University, 138 Yixueyuan Road, Shanghai 200032, P.R. China

E-mail: yuqiuzhang@fudan.edu.cn

*Contributed equally

Key words: dexmedetomidine, anti-nociceptive, inflammatory cytokine, signaling pathway injection. These results suggested that DEX has an anti-nociceptive effect via suppressing the TLR4/NF-кB p65 pathway.

\section{Introduction}

Arthritis is a common medical problem associated with chronic pain, which may further cause a pain syndrome characterized by spontaneous pain, allodynia and hyperalgesia. Joint inflammation inducing peripheral and central sensitization is thought to be the main cause of arthritic pain $(1,2)$, and thus, targeting joint inflammation may be a promising therapeutic strategy for the treatment of arthritis.

Dexmedetomidine (DEX) is a highly selective agonist of $\alpha 2$-adrenoceptors ( $\alpha 2 \mathrm{AR})$ and has been found to bind $\alpha 2 \mathrm{AR}$ more avidly than clonidine (3), which has demonstrated significant analgesic effects on acute inflammatory pain (2), post-operative pain (4) and neuropathic pain unresponsive to opioid analgesics $(5,6)$. In addition, various $\alpha 2 \mathrm{AR}$ agonists have been demonstrated to have anti-nociceptive properties, and the roles of $\alpha 2 \mathrm{AR}$ in pain regulation have been widely investigated in the spinal cord (7-9).

Toll-like receptor 4 (TLR4), a member of the TLR family, is an important transmembrane protein involved in signal transduction. Previous studies have demonstrated that TLR4 participates in the defense against Gram-negative bacteria, and lipopolysaccharide (LPS) is its most common ligand (10). The downstream signaling molecules of TLR4 mainly include nuclear factor $(\mathrm{NF})-\kappa \mathrm{B}$, an important transcriptional factor (11). The activated $\mathrm{NF}-\kappa \mathrm{B}$ protein enters into the nucleus, initiating the transcription of numerous genes, including the pro-inflammatory tumor necrosis factor (TNF)- $\alpha$, interleukin (IL)-1 $\beta$ and IL-6 (12).

Spinal glia such as astrocytes and microglia, which are central nervous system (CNS)-specific tissue macrophages, have been demonstrated to have central roles in chronic pain development and maintenance (13-15). For instance, spinal glia activation has been implicated in the behavioral hypersensitivity induced by spinal injury, peripheral nerve injury as well as contact with formalin, zymosan and complete Freund's adjuvant (CFA) (16-21). In addition, fluorocitrate- or minocycline-induced destruction of glial function significantly attenuated mechanical allodynia and thermal hyperalgesia 
in CFA-induced monoarthritic (MA) rats (19-22). Therefore, activated glial cells are suggested to be involved in joint inflammation-induced behavioral hypersensitivity.

Similar to that of neurons, the membrane of glial cells expresses $\alpha 2 \mathrm{ARs}$ in the CNS (23-25), but the function of $\alpha 2 \mathrm{ARs}$ in glial cells has remained largely elusive. The expression of glial fibrillary acid protein (GFAP) has been demonstrated to be functionally linked to $\alpha 2 \mathrm{ARs}$ in astrocytes (26). However, the molecular mechanism underlying the anti-nociceptive effects of DEX on astrocytes has not been fully uncovered.

The present study aimed to investigate whether repeated intrathecal injection with DEX has anti-nociceptive effects through inhibition of TLR4 expression, NF- $\kappa \mathrm{B}$ activation and pro-inflammatory cytokine production in rats with CFA-induced ankle joint MA.

\section{Materials and methods}

Reagents. DEX was purchased from Jiangsu Hengrui Medicine (Lianyungang, China). VonFrey hairs were purchased from Stoelting (Wood Dale, IL, USA). BRL44408, CFA, rabbit anti-NF- $\kappa$ B p65 primary antibody (cat. no. SAB4502610), rabbit anti- $\alpha 2 A R$ primary antibody (cat. no. A271) and mouse anti-GFAP primary antibody (cat. no. SAB1405864) were purchased from Sigma-Aldrich (Merck KGaA, Darmstadt, Germany). Donkey anti-rabbit immunoglobulin G (IgG; cat. no. 711-005-152) and donkey anti-mouse IgG (cat. no. 715-005-150) were purchased from Jackson ImmunoResearch (West Grove, PA, USA). Rabbit anti-TLR4 primary antibody (cat. no. ab13556) was purchased from Abcam (Cambridge, MA, USA). Rabbit anti NF- $\kappa$ B p65 antibody (cat. no. 8242) and rabbit anti GAPDH antibody (cat. no. 2118) were purchased from Cell Signaling Technology, Inc. (Danvers, MA, USA). Horseradish peroxidase-conjugated donkey anti-mouse secondary antibody (cat. no. sc-2318) was purchased from Santa Cruz Biotechnology, Inc. (Dallas, TX, USA). A bicinchoninic acid (BCA) kit, polyvinylidene difluoride (PVDF) membrane, goat anti-rabbit secondary antibody (cat. no. 65-6120) and Super Signal West Pico Chemiluminescent Substrate kit (cat. no. 34080) were purchased from Thermo Fisher Scientific, Inc. (Waltham, MA, USA).

Animals. The present study was approved by the Animal Care and Use Committee of Fudan University (Shanghai, China), and all animal experiments were consistent with the National Institutes of Health Guide for the Care and Use of Laboratory Animals. A total of 40 adult Sprague Dawley rats (male; weight, 200-250 g; 3 months old) were purchased from the Experimental Animal Center of Fudan University (Shanghai, China). These rats were housed in a temperature- $\left(22 \pm 2^{\circ} \mathrm{C}\right)$ and light-controlled (12-h light/dark cycle) room with free access to food and water.

The rats were randomly divided into 5 groups of 8 animals each: The Sham group [intra-articular injection with $50 \mu 1$ sterile saline and intrathecal (i.t.) injection with $20 \mu \mathrm{l}$ sterile saline for 3 consecutive days], the CFA group (intra-articular injection with $50 \mu$ l CFA to induce MA), the DEX group [intra-articular injection with $50 \mu \mathrm{l} \mathrm{CFA}$ and intrathecal injection with $\operatorname{DEX}(2.5 \mu \mathrm{g} / 20 \mu \mathrm{l})$ for 3 consecutive days post-MA], the B group [intra-articular injection with $50 \mu \mathrm{l} \mathrm{CFA} \mathrm{and}$ pre-treatment with BRL44408 $(15 \mu \mathrm{g} / 20 \mu \mathrm{l})$ for $30 \mathrm{~min}$ prior to i.t. injection with $\operatorname{DEX}(2.5 \mu \mathrm{g} / 20 \mu \mathrm{l})$ for 3 consecutive days post-MA], the epigallocatechin gallate (EGCG) group (intra-articular injection with $50 \mu \mathrm{l} \mathrm{CFA}$ and intrathecal injection with $30 \mu \mathrm{g}$ EGCG for 3 consecutive days post-MA). The Sham, DEX, B and EGCG groups were treated by i.t. injection on 3 consecutive days after MA, and the pain test was performed $1 \mathrm{~h}$ after the injection.

Drug administration. DEX $(2.5 \mu \mathrm{g} / 20 \mu \mathrm{l})$ and BRL44408 $(15 \mu \mathrm{g} / 20 \mu \mathrm{l})(27)$ were diluted in normal saline (NS, $0.9 \%$ $\mathrm{NaCl}$ ). Lumbar puncture (LP) injection was performed as previously described (28). Under inhalation anesthesia using isoflurane (2\% in oxygen; Abbott Pharmaceutical Co. Ltd., Lake Bluff, IL, USA), the rats were injected at the L5-6 interspace. The occurrence of an instantaneous and rapid tail-flick indicated a successful puncture. Control animals received an equivalent volume of sterile saline. Animals then recovered in their cage prior to analgesic testing.

Induction of MA. Isoflurane was used to anesthetize the rats, and an iodine tincture and $75 \%$ alcohol were used to sterilize the skin. The fossa of the lateral malleolus of the fibula was located, and a 28-gauge needle was inserted from the gap between the tibiofibular and tarsus bone into the articular cavity. CFA $(50 \mu \mathrm{l})$ was injected into the articular cavity. The Sham MA group was injected with sterile saline.

Von Frey test for mechanical allodynia. Mechanical allodynia was assessed by examining the paw withdrawal mechanical threshold (PWMT) in response to a calibrated series of Von Frey hairs (29). Each filament was applied five times, each application lasted for $2 \mathrm{sec}$, and there was a 30-sec interval between each application. A positive response to a filament was indicated by the withdrawal of a hind paw upon application of a particular hair for at least 3 out of 5 consecutive applications. The PWT was defined as the smallest value of the hair force in grams that elicited positive responses.

Hargreaves' test for thermal hyperalgesia. Thermal hyperalgesia was studied by examining the paw withdrawal thermal latency (PWTL) in response to a radiant heat source. Rats in each group were put on an elevated glass platform. A radiant heat source (Model 336; IITC Life Science, Woodland Hill, CA, USA) was then applied to the plantar surface of the hind paw through the glass plate. The time from onset of radiant heat application to withdrawal of the hind paw was measured, and both hind paws were tested independently with a 10 -min interval between trials $(19,30)$.

Immunohistochemistry. Rats were given urethane $(2 \mathrm{~g} / \mathrm{kg}$, intraperitoneally) and perfused with $4 \%$ paraformaldehyde in $0.1 \mathrm{~mol} / 1$ phosphate buffer through the ascending aorta. Subsequently, the L4-5 segments of the spinal cord were fixed in $4 \%$ paraformaldehyde at $4^{\circ} \mathrm{C}$ for $4 \mathrm{~h}$, which were then immersed in sucrose (10-30\%) for $24 \mathrm{~h}$ at $4^{\circ} \mathrm{C}$. Sections (35 $\mu \mathrm{m})$ were cut, which were then blocked in PBS with $10 \%$ donkey serum (cat. no. ab7475; Abcam) and 0.3\% Triton X-100 at room temperature (RT) for $2 \mathrm{~h}$. Subsequently, the sections were incubated overnight at $4^{\circ} \mathrm{C}$ with rabbit anti-NF- $\kappa \mathrm{B}$ p65 
(1:200 dilution) or mouse anti-GFAP (1:2,000 dilution) primary antibody in PBS with $1 \%$ donkey serum and $0.3 \%$ Triton $\mathrm{X}-100$. After washing with PBS for 3 times, the sections were incubated in donkey anti-rabbit $\operatorname{IgG}$ (1:200 dilution) for NF- $\mathrm{B}$ p65 or donkey anti-mouse IgG (1:200 dilution) for GFAP for $2 \mathrm{~h}$ at $4^{\circ} \mathrm{C}$. The sections were observed with a fluorescence microscope (Leica Microsystems, Wetzlar Germany).

Immunofluorescence. We chose the CFA group at 3 days to detect the double immunofluorescence. Spinal sections were incubated with a mixture of rabbit anti-TLR4 (1:500 dilution) and mouse anti-GFAP (1:2,000 dilution), rabbit anti- $\alpha 2 \mathrm{AR}$ (1:2,000 dilution) and mouse anti-GFAP (1:2,000 dilution) overnight at $4^{\circ} \mathrm{C}$, followed by a mixture of donkey anti-rabbit $\operatorname{IgG}(1: 200$ dilution) for TLR4 and $\alpha 2 \mathrm{AR}$ and donkey anti-mouse $\operatorname{IgG}$ (1:200 dilution) for GFAP for $1 \mathrm{~h}$ at RT. The sections were observed with a fluorescence microscope (Leica Microsystems).

Western blot analysis. The L4-5 segments of spinal cords were dissected and split into left and right halves from the ventral midline, which were then cut into the dorsal and ventral horn at the level of the central canal. The dorsal horn was then lysed with ice-cold lysis buffer. Proteins $(50 \mu \mathrm{g})$ in the supernatants were quantified by using a BCA kit and separated by $12 \%$ SDS-PAGE. Proteins were transferred onto a PVDF membrane, which was then incubated with PBS containing $5 \%$ milk overnight at $4^{\circ} \mathrm{C}$. Subsequently, the membrane was incubated with mouse anti-GFAP (1:2,000 dilution), rabbit anti-TLR4 (1:1,000 dilution), rabbit anti NF-кB p65 (1:1,000 dilution) and GAPDH (1:20,000 dilution) antibodies overnight at $4^{\circ} \mathrm{C}$, and then with horseradish peroxidase-conjugate donkey anti-mouse secondary antibody (1:2,000 dilution) and goat anti-rabbit secondary antibody (1:4,000 dilution) for $2 \mathrm{~h}$ at RT. Signals on the membrane were visualized using the Super Signal West Pico Chemiluminescent Substrate kit and exposed to X-ray films (Bio-Rad Laboratories, Inc., Hercules, CA, USA) for 1-10 min as follows: GFAP $5 \mathrm{~min}$, TLR4 $8 \mathrm{~min}$, NF- $\mathrm{B}$ p65 $10 \mathrm{~min}$ and GAPDH $3 \mathrm{~min}$. The optical densities of bands were analyzed by Image-Pro plus software 6.0 (Media Cybernetics, Inc., Rockville, MD, USA) and presented as the density ratio vs. GAPDH.

ELISA. ELISA was performed to examine the levels of IL-1 $\beta$, IL- 6 and TNF- $\alpha$ in the L4-5 dorsal horns of the MA side using the Rat IL-1 $\beta$ (cat. no. ab100768), IL-6 (cat. no. ab100772) and TNF- $\alpha$ (cat. no. ab100785) ELISA kits, according to the manufacturer's protocols.

Co-immunoprecipitation. Protein G Sepharose (10 $\mu \mathrm{l})$ was washed with lysate buffer for 3 times, followed by centrifugation at 3,000 x g for 3 min once. A total of $20 \mu \mathrm{g}$ sample protein preparation from the western blot experiment was added to the Protein $\mathrm{G}$ Sepharose and incubated overnight at $4^{\circ} \mathrm{C}$ with gentle shaking. Protein from monoarthritic rats of 3 days as the positive control, the normal IgG protein as the negative control. Next, after centrifugation at 3,000 x $\mathrm{g}$ for $3 \mathrm{~min}$ at $4^{\circ} \mathrm{C}$, the supernatant was carefully removed and the Protein G Sepharose was washed with $1 \mathrm{ml}$ lysate buffer for 3 times. A total of $6 \mu \mathrm{l}$ sample buffer was added, followed by boiling at $70^{\circ} \mathrm{C}$. Finally, the samples were subjected to western blot analysis.

Statistical analysis. We used the Sigmastat 3.1 (Systat software, Inc., San Jose, CA, USA) for statistical analysis. Values are expressed as the mean \pm standard error of the mean. One-way analysis of variance (ANOVA) was used to analyze the pre-MA baseline and pre-drug treatment measures. Two-way ANOVA (treatment time) followed by the Holm-Sidak test was used to analyze the post-drug time course measures for hyperalgesia. Immunohistochemical and western blot analysis results were analyzed by Student's t-test when comparing two groups or one-way ANOVA followed by the Holm-Sidak test when comparing $>2$ groups. $\mathrm{P}<0.05$ was considered to indicate a statistically significant difference.

\section{Results}

Effects of DEX treatment on GFAP expression as well as mechanical and thermal hypersensitivity in rats with CFA-induced MA. As presented in Fig. 1A and B, the measures of PWMT and PWTL in either hind paw in the sham group did not differ prior to and after intra-articular saline injection; however, unilateral intra-articular injection with CFA caused significant mechanical or thermal hyperalgesia, which peaked at 3 days after CFA injection. Mechanical and thermal hyperalgesia significantly increased in the CFA group compared with the control group ( $\mathrm{P}<0.01$; Fig. $1 \mathrm{~A}$ and $\mathrm{B})$.

Furthermore, the effects of repeated injection with DEX on PWMT or PWTL different time-points after intra-articular injection with CFA were determined. DEX or sterile saline were given i.t. for 3 consecutive days after MA. Compared with that in the CFA group, the PWMT in the DEX group was significantly increased at $1,3,5$ and 7 days ( $\mathrm{P}<0.01$; Fig. 1A). Furthermore, the PWTL was enhanced in the DEX group at $1,3,5$ and 7 days $(\mathrm{P}<0.01)$, as well as in the EGCG group compared with that in the CFA group (Fig. 1B). In comparison with that in the B group, the PWMT and PWTL in the DEX and in the EGCG group were significantly enhanced at 1, 3, 5 and 7 days $(\mathrm{P}<0.01$; Fig. $1 \mathrm{~A}$ and $\mathrm{B})$, which indicated that pre-treatment with BRL44408 for 3 consecutive days abrogated the beneficial effect of DEX.

Following CFA-induced MA, the protein expression of GFAP was detected by western blot analysis at 3 and 7 days. The results demonstrated that the expression of GFAP was significantly increased in the ipsilateral spinal dorsal horn of the CFA at 3 and 7 days, which was suppressed by intrathecal injection with DEX and EGCG for 3 consecutive days, and pre-treatment with BRL44408 for 3 consecutive days abrogated the effect of DEX (Fig. 1C and D). These findings suggested that the upregulation of GFAP may be associated with the pain threshold.

TLR4 expression and $N F-\kappa B$ p 65 activity in the spinal dorsal horn. Following CFA-induced MA, western blot analysis was performed to examine the protein levels of TLR4 and $\mathrm{NF}-\kappa \mathrm{B}$ p65 at 3 and 7 days. The results indicated that, when compared with the sham group, the TLR4 levels were significantly increased in the ipsilateral spinal dorsal horn of the CFA group at $3(\mathrm{P}=0.0001)$ and $7(\mathrm{P}=0.00021)$ days; 


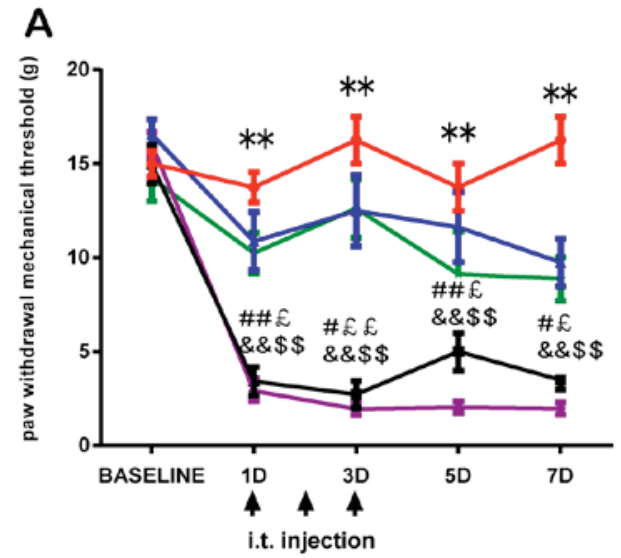

Time after CFA injection

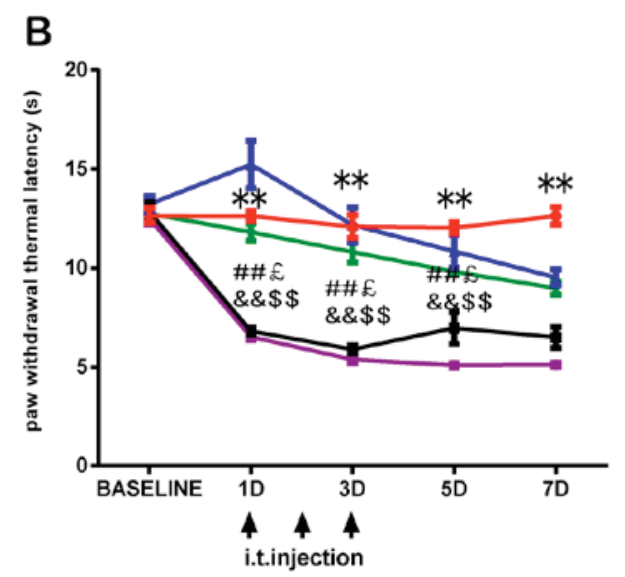

Time after CFA injection (d)

D
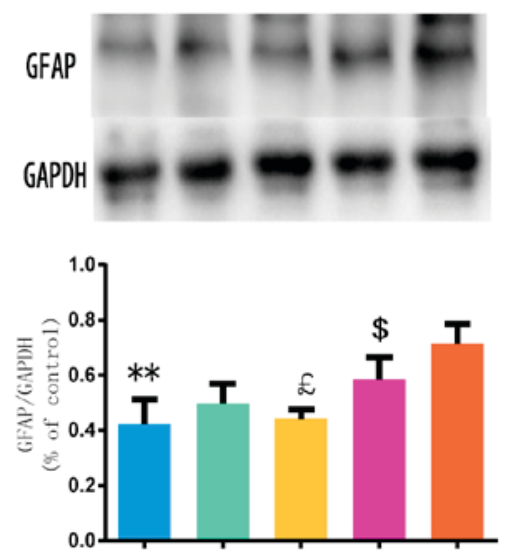

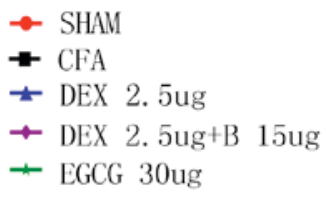

\section{C}
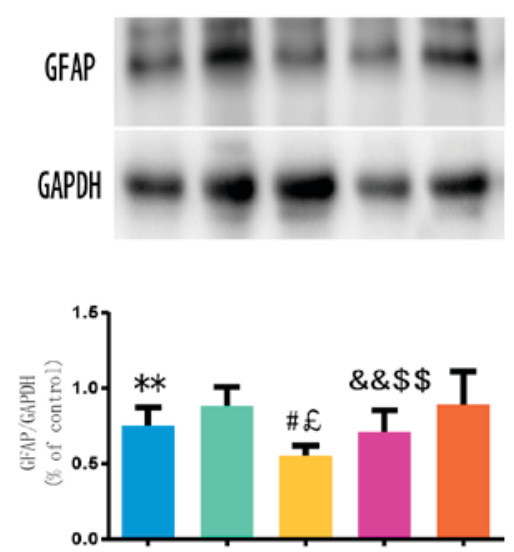

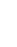

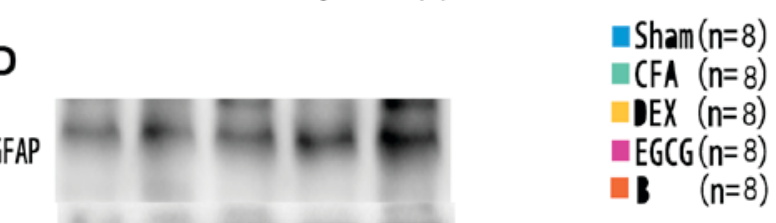

Figure 1. Expression of GFAP and the development of mechanical and thermal hypersensitivity in CFA-induced MA. Effects of i.t. injection with DEX (2.5 $\mu \mathrm{g})$ on (A) paw withdrawal mechanical threshold and (B) paw withdrawal thermal latency of the ipsilateral hind paws prior to the behavioral test on day 1-3 after surgery. ${ }^{* *} \mathrm{P}<0.05$, SHAM vs. CFA; ${ }^{\#} \mathrm{P}<0.01,{ }^{\# \#} \mathrm{P}<0.05$, CFA vs. DEX; ${ }^{£} \mathrm{P}<0.01,{ }^{£ f} \mathrm{P}<0.05, \mathrm{CFA}$ vs. EGCG; \&\& P<0.05, B vs. DEX; ${ }^{\$ \$} \mathrm{P}<0.05, \mathrm{~B}$ vs. EGCG. $(\mathrm{C}$ and D) Western blot analysis of GFAP in the spinal dorsal horn of rats in the Sham, CFA, DEX, EGCG and B groups. Effects of intrathecal injection with DEX, BRL44408 and EGCG over 3 consecutive days on the GFAP levels in the spinal dorsal horn induced by MA on (C) day 3 and (D) day 7. GAPDH served as loading control. ${ }^{* *} \mathrm{P}<0.05$, SHAM vs. CFA; ${ }^{\#} \mathrm{P}<0.01$, DEX vs. CFA; ${ }^{\circledR} \mathrm{P}<0.01$, DEX vs. B; ${ }^{\&} \mathrm{P}<0.05$, EGCG vs. CFA; ${ }^{\$} \mathrm{P}<0.01,{ }^{\$ \$} \mathrm{P}<0.05, \mathrm{EGCG}$ vs. B. Groups: Sham, i.a. injection with $50 \mu 1$ sterile saline and i.t. injection with $20 \mu 1$ sterile saline for 3 consecutive days; CFA, i.a. injection with 50 $\mu$ l CFA; DEX, i.a. injection with $50 \mu \mathrm{l} \mathrm{CFA}$ and i.t. injection with DEX $(2.5 \mu \mathrm{g} / 20 \mu \mathrm{l})$ for 3 consecutive days; B, i.a. injection with $50 \mu \mathrm{l} \mathrm{CFA}$ and pre-treatment with BRL44408 $(15 \mu \mathrm{g} / 20 \mu \mathrm{l}) 30 \mathrm{~min}$ prior to i.t. injection with DEX $2.5(\mu \mathrm{g} / 20 \mu \mathrm{l})$ for 3 consecutive days; EGCG, i.a. injection with $50 \mu \mathrm{l}$ CFA and i.t. injection with EGCG $30 \mu \mathrm{g}$ for 3 consecutive days. CFA, complete Freund's adjuvant; MA, monoarthritis; DEX, dexmedetomidine; i.t., intrathecal; i.a., intra-articular; EGCG, epigallocatechin gallate; B, BRL44408; GFAP, glial fibrillary acid protein.

when compared with the CFA group at 3 and 7 days, the TLR4 levels were suppressed by intrathecal injection with $\operatorname{DEX}(\mathrm{P}=0.0007$ and $\mathrm{P}=0.0015$, respectively) and $\mathrm{EGCG}$ $\left(P_{3 \mathrm{~d}}=0.00082\right.$ and $\left.\mathrm{P}_{7 \mathrm{~d}}=0.0003\right)$ for 3 consecutive days; when compared with the B group at 3 day, the TLR4 levels were suppressed by intrathecal injection with DEX $(\mathrm{P}=0.0001)$ and EGCG $(\mathrm{P}=0.00022)$. Furthermore, pretreatment with BRL44408 for 3 consecutive days abrogated the effect of DEX (Fig. 2A). Furthermore, when compared with the sham group, NF- $\kappa \mathrm{B}$ p65 levels were significantly increased in the ipsilateral spinal dorsal horn of the CFA and B groups at 3 and 7 days, which was suppressed by intrathecal injection with DEX and EGCG for 3 consecutive days, and pretreatment with BRL44408 for 3 consecutive days abrogated the effect of DEX (Fig. 2B). These findings suggested that the upregulation of TLR4 and NF- $\mathrm{B}$ p 65 may also be associated with the pain thresholds.
Immunohistochemical analysis of $N F-\kappa B$ p 65 in the spinal dorsal horn. After the pain test at 3 days after MA, 3 rats in each group were sacrificed and immunohistochemistry was performed to examine the expression of $\mathrm{NF}-\kappa \mathrm{B}$ p65. As indicated in Fig. 3, NF- $\mathrm{B}$ p65-positive cells were distributed in the spinal dorsal horn. In addition, the CFA group contained a markedly higher amount of positive staining for NF- $\mathrm{B}$ p 65 in the spinal dorsal horn compared with that in the sham group, while treatment with DEX and EGCG significantly reduced the expression of NF- $\mathrm{B}$ p65 at 3 days after MA (Fig. 3).

Levels of $I L-1 \beta, I L-6$ and TNF- $\alpha$ in the spinal dorsal horn. The contents of IL-1 $\beta, T N F-\alpha$ and IL- 6 were determined by ELISA. The results demonstrated that, when compared with the Sham group, the contents of IL- $1 \beta\left(\mathrm{P}_{3 \mathrm{~d}}=0.006\right.$ and $\left.\mathrm{P}_{7 \mathrm{~d}}=0.005\right)$, TNF- $\alpha\left(\mathrm{P}_{3 \mathrm{~d}}=0.002\right.$ and $\left.\mathrm{P}_{7 \mathrm{~d}}=0.012\right)$ and IL-6 $\left(\mathrm{P}_{3 \mathrm{~d}}=0.0024\right.$ and $\left.\mathrm{P}_{7 \mathrm{~d}}=0.0022\right)$ increased significantly in the CFA group. When 
A
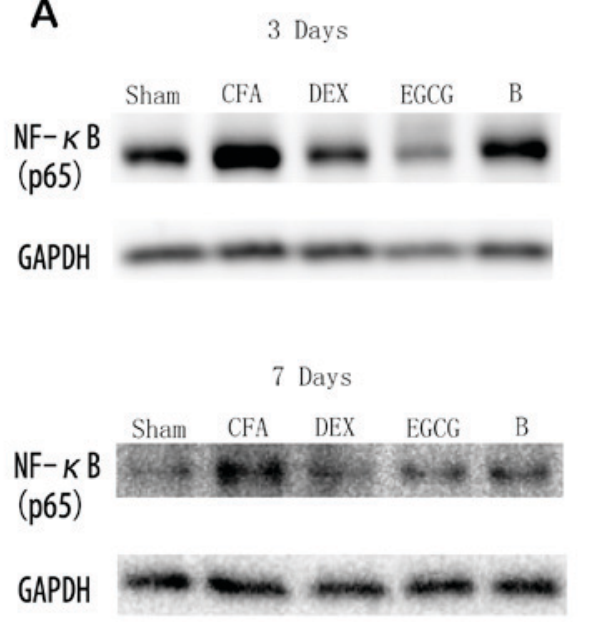

B
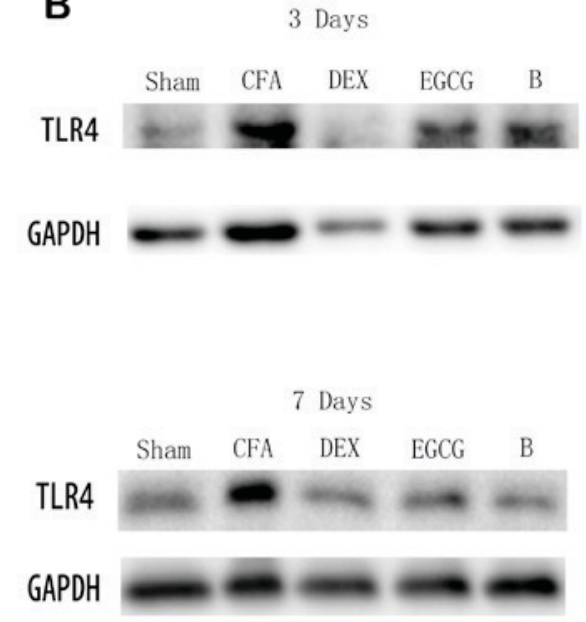
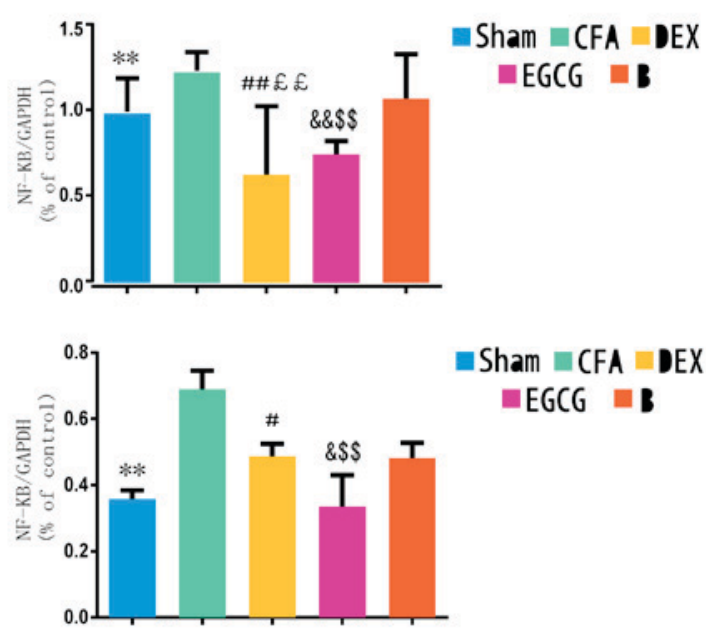

"Sham $=$ CFA $=$ DEX

EGCG $\mathbf{B}$

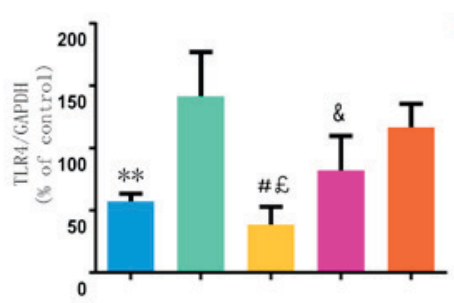

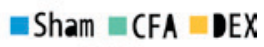

EGCG $\mathbf{B}$

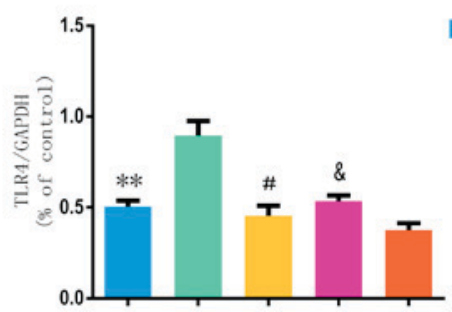

nSham "CFA DEX

口EGG $\mathbf{B}$

Figure 2. Western blot analysis of TLR4 expression and NF-kB p65 activity in spinal dorsal horn of Sham, CFA, DEX, EGCG and B groups. (A and B) Effects of intrathecal injection with DEX, BRL44408 and EGCG for 3 consecutive days on the (A) NF- $\mathrm{kB}$ p65 and (B) TLR4 levels in the spinal dorsal horn induced by MA on days 3 and 7 after MA, respectively. GAPDH served as loading control. ${ }^{* *} \mathrm{P}<0.05$, SHAM vs. CFA; ${ }^{\#} \mathrm{P}<0.01$ and ${ }^{\# \#} \mathrm{P}<0.05$, DEX vs. CFA; ${ }^{\text {I }} \mathrm{P}<0.01$ and ${ }^{\text {ff }} \mathrm{P}<0.05$, DEX vs. $\mathrm{B} ;{ }^{\&} \mathrm{P}<0.01$ and ${ }^{\& \&} \mathrm{P}<0.05$, EGCG vs. CFA; ${ }^{\$} \mathrm{P}<0.01$ and ${ }^{\$ \$} \mathrm{P}<0.05$, EGCG vs. B. Sham, i.a. injection with $50 \mu 1$ sterile saline and i.t. injection with $20 \mu \mathrm{l}$ sterile saline for 3 consecutive days; CFA, i.a. injection with $50 \mu \mathrm{l} \mathrm{CFA}$; DEX, i.a. injection with $50 \mu \mathrm{l} \mathrm{CFA}$ and i.t. injection with DEX (2.5 $\mu \mathrm{g} / 20 \mu \mathrm{l})$ for 3 consecutive days; B, i.a. injection with $50 \mu \mathrm{l} \mathrm{CFA}$ and pre-treatment with BRL44408 $(15 \mu \mathrm{g} / 20 \mu \mathrm{l}) 30$ min prior to i.t. injection with DEX $2.5(\mu \mathrm{g} / 20 \mu \mathrm{l})$ for 3 consecutive days; EGCG, i.a. injection with $50 \mu \mathrm{l} \mathrm{CFA}$ and i.t. injection with EGCG $30 \mu \mathrm{g}$ for 3 consecutive days. i.t., intrathecal; i.a., intra-articular; TLR, Toll-like receptor; NF, nuclear factor; CFA, complete Freund's adjuvant; MA, monoarthritis; DEX, dexmedetomidine; EGCG, epigallocatechin gallate; B, BRL44408.

compared with the CFA and B groups, the expression of IL-1 $\beta$ was markedly decreased in the DEX (vs. CFA, $\mathrm{P}_{3 \mathrm{~d}}=0.0001$ and $\mathrm{P}_{7 \mathrm{~d}}=0.00022$; vs. $\mathrm{B}, \mathrm{P}_{3 \mathrm{~d}}=0.0001$ and $\left.\mathrm{P}_{7 \mathrm{~d}}=0.0003\right)$ group at 3 and 7 days, as well as in the EGCG (vs. CFA, $P_{3 \mathrm{~d}}=0.0007$ and $\mathrm{P}_{7 \mathrm{~d}}=0.0003$; vs. $\mathrm{B}, \mathrm{P}_{3 \mathrm{~d}}=0.0001$ and $\mathrm{P}_{7 \mathrm{~d}}=0.015$ ) group at 3 and 7 days, (Fig. 4A). The expression of TNF- $\alpha$ was significantly decreased in the DEX (vs. CFA, $\mathrm{P}_{3 \mathrm{~d}}=0.0002$ and $\mathrm{P}_{7 \mathrm{~d}}=0.00034$; vs. $\mathrm{B}, \mathrm{P}_{3 \mathrm{~d}}=0.00016$ and $\mathrm{P}_{7 \mathrm{~d}}=0.0009$ ) group at 3 and 7 days, as well as in the EGCG (vs. CFA, $\mathrm{P}_{3 \mathrm{~d}}=0.00012$ and $\mathrm{P}_{7 \mathrm{~d}}=0.015$; vs. $\mathrm{B}, \mathrm{P}_{3 \mathrm{~d}}=0.00026$ and $\mathrm{P}_{7 \mathrm{~d}}=0.03$ ) group at 3 and 7 days, when compared with the CFA and $\mathrm{B}$ group at 3 and 7 days (Fig. 4B). Compared with that in the CFA group, the content of IL-6 was significantly decreased in the DEX $\left(\mathrm{P}_{3 \mathrm{~d}}=0.0007\right.$ and $\left.\mathrm{P}_{7 \mathrm{~d}}=0.0022\right)$ and EGCG $\left(\mathrm{P}_{3 \mathrm{~d}}=0.0001\right.$ and $\left.\mathrm{P}_{7 \mathrm{~d}}=0.018\right)$ groups at 3 and 7 days; furthermore, IL- 6 in the $\mathrm{B}$ group at $3(\mathrm{P}=0.0004)$ and $7(\mathrm{P}=0.012)$ days was significantly higher than that in the DEX group (Fig. 4C).
Double immunofluorescence of GFAP and TLR4 and $\alpha 2 A R$ with GFAP in the spinal dorsal horn, and the interaction between $\alpha 2 A R$ and TLR4. We chose the CFA group at 3 days to detect the double immunofluorescence. TLR4 and $\alpha 2 A R$ immunoreactivity co-localized with GFAP, indicating that TLR4 and $\alpha 2 \mathrm{AR}$ were expressed in the same cell of the spinal dorsal horn (Fig. 5A). The above results demonstrated that i.t. administration of DEX for 3 consecutive days not only attenuated hyperalgesia, but also inhibited TLR4 expression and NF- $\kappa \mathrm{B}$ p 65 activity in the spinal cord. Therefore, it was speculated that $\alpha 2 \mathrm{AR}$ possibly interacts with TLR4 via a certain unknown mechanism. Therefore, the present study first detected whether $\alpha 2 \mathrm{AR}$ and TLR4 were expressed in the same cell of the spinal dorsal horn. Immunofluorescence confirmed that $\alpha 2 \mathrm{AR}$ and TLR4 were significantly co-expressed in astrocytes of the spinal dorsal horn, which provided possible histological evidence for the interaction between $\alpha 2 \mathrm{AR}$ and TLR4. Furthermore, the interaction 

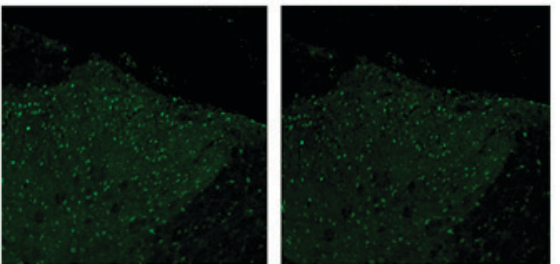

Sham
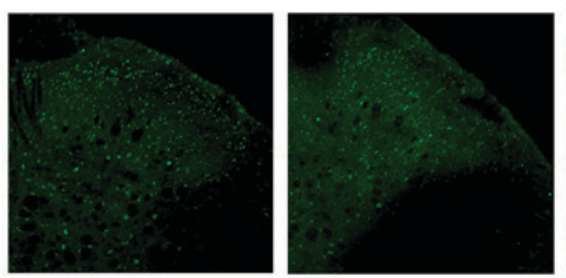

DEX

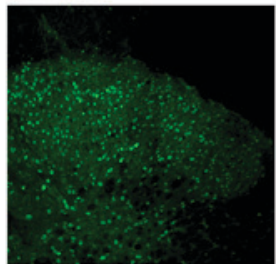

CFA
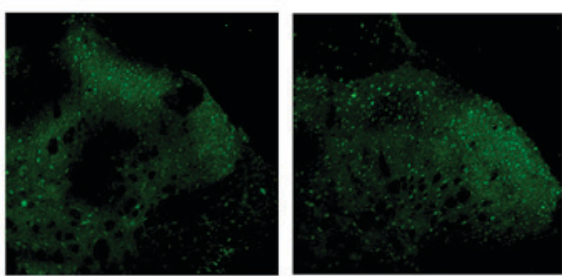

EGCG

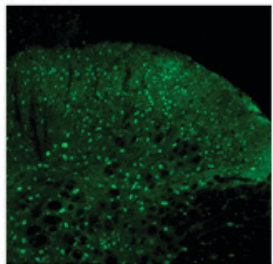

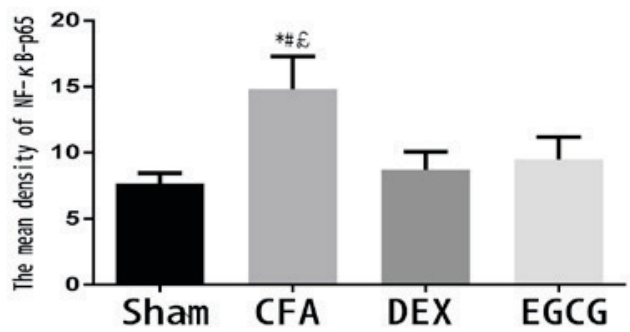

Figure 3. Activation of NF-kB p65 in the spinal dorsal horn on day 3 after MA in the Sham, CFA, DEX and EGCG groups. Immunohistochemistry revealed

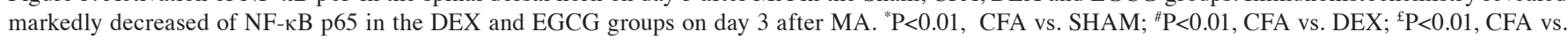
EGCG. Magnification, x20. Sham, i.a. injection with $50 \mu 1$ sterile saline and i.t. injection with $20 \mu 1$ sterile saline for 3 consecutive days; CFA, i.a. injection with $50 \mu \mathrm{l}$ CFA; DEX, i.a. injection with $50 \mu \mathrm{l}$ CFA and i.t. injection with DEX $(2.5 \mu \mathrm{g} / 20 \mu \mathrm{l})$ for 3 consecutive days; EGCG, i.a. injection with $50 \mu \mathrm{l}$ CFA and i.t. injection with EGCG $30 \mu \mathrm{g}$ for 3 consecutive days. i.t., intrathecal; i.a., intra-articular; NF, nuclear factor; CFA, complete Freund's adjuvant; MA, monoarthritis; DEX, dexmedetomidine; EGCG, epigallocatechin gallate.

A

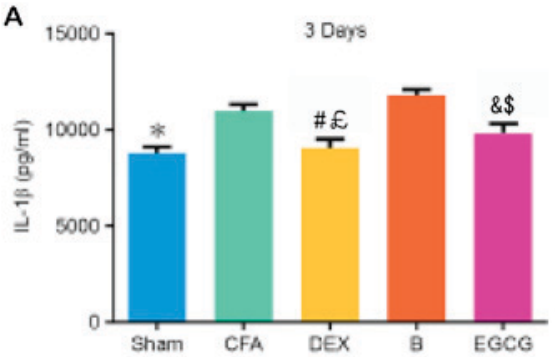

B

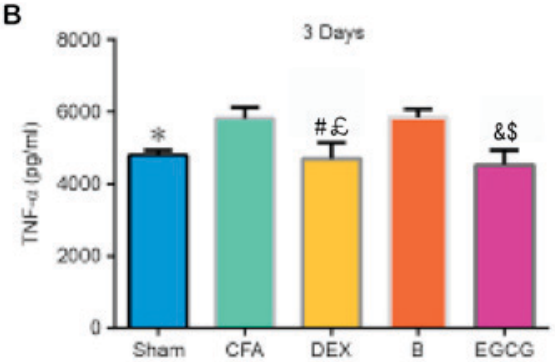

C

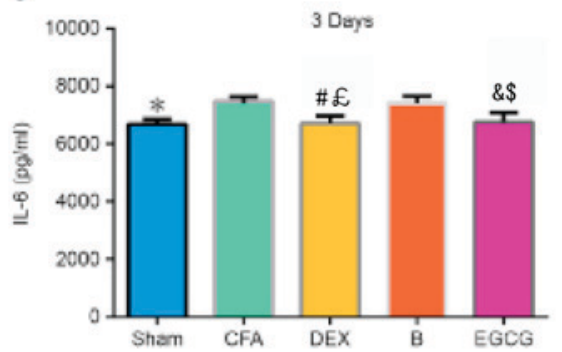

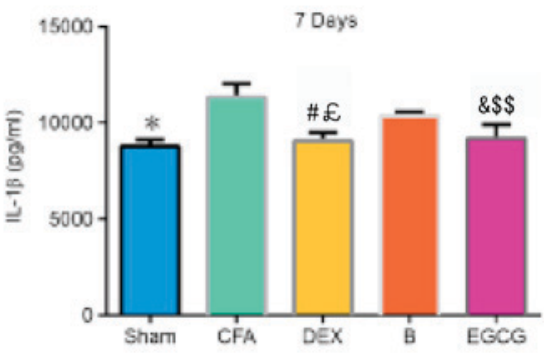
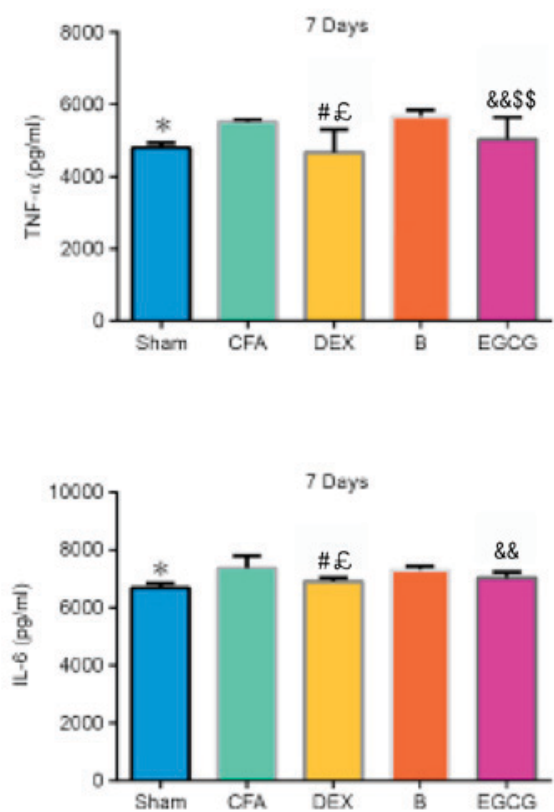

Figure 4. Effects of intrathecal administration of DEX, BRL44408 or EGCG on the expression of (A) IL-1 $\beta$, (B) TNF- $\alpha$ and (C) IL-6 in the L4-5 dorsal horns of the MA side of MA rats. Values are expressed as the mean \pm standard deviation. ${ }^{*} \mathrm{P}<0.01$, SHAM vs. CFA; ${ }^{\text {P }}<0.01$, DEX vs. CFA; ${ }^{\text {I}} \mathrm{P}<0.01$, DEX vs. $\mathrm{B}$; ${ }^{\&} \mathrm{P}<0.01,{ }^{\&}{ }^{\&} \mathrm{P}<0.05$, EGCG vs. CFA; ${ }^{\$} \mathrm{P}<0.01,{ }^{\$} \mathrm{P}<<0.05$, EGCG vs. B. Groups: Sham, i.a. injection with $50 \mu 1$ sterile saline and i.t. injection with $20 \mu 1$ sterile saline for 3 consecutive days; CFA, i.a. injection with $50 \mu \mathrm{l} \mathrm{CFA;} \mathrm{DEX,} \mathrm{i.a.} \mathrm{injection} \mathrm{with} 50 \mu \mathrm{l} \mathrm{CFA}$ and i.t. injection with DEX $(2.5 \mu \mathrm{g} / 20 \mu \mathrm{l})$ for 3 consecutive days; B, i.a. injection with $50 \mu \mathrm{l}$ CFA and pre-treatment with BRL44408 $(15 \mu \mathrm{g} / 20 \mu \mathrm{l}) 30 \mathrm{~min}$ prior to i.t. injection with DEX $2.5(\mu \mathrm{g} / 20 \mu \mathrm{l})$ for 3 consecutive days; EGCG, i.a. injection with $50 \mu \mathrm{l} \mathrm{CFA}$ and i.t. injection with EGCG $30 \mu \mathrm{g}$ for 3 consecutive days. i.t., intrathecal; i.a., intra-articular; CFA, complete Freund's adjuvant; MA, monoarthritis; DEX, dexmedetomidine; EGCG, epigallocatechin gallate; B, BRL44408; IL, interleukin; TNF, tumor necrosis factor. 
A

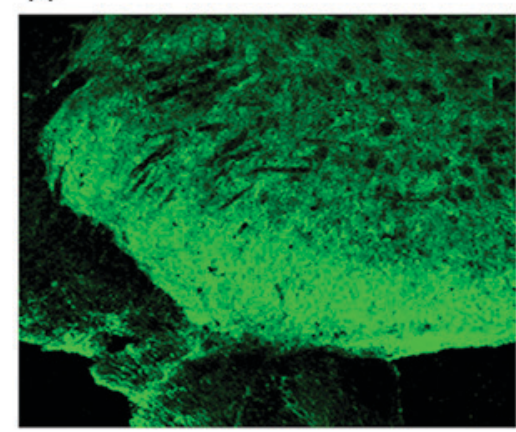

$\alpha 2 \mathrm{AR}$

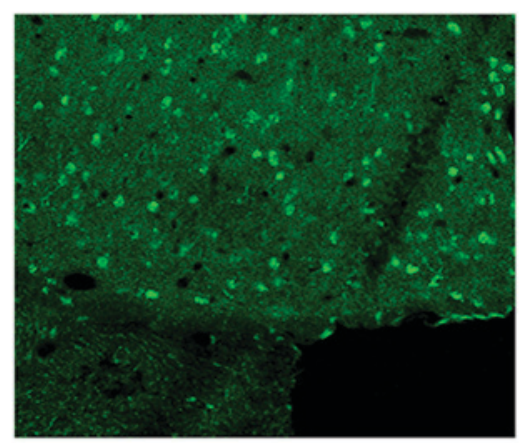

TLR4

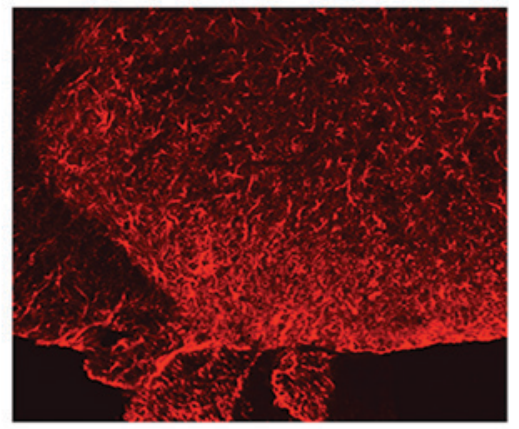

GFAP

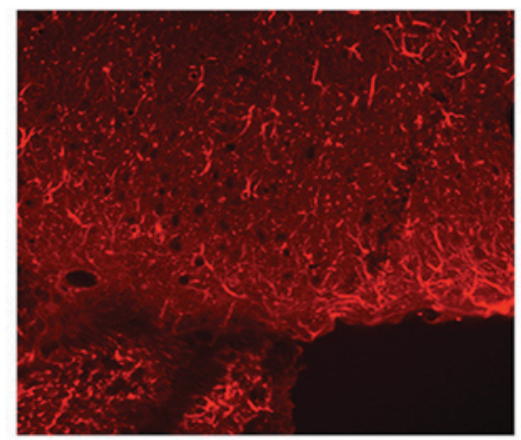

GFAP

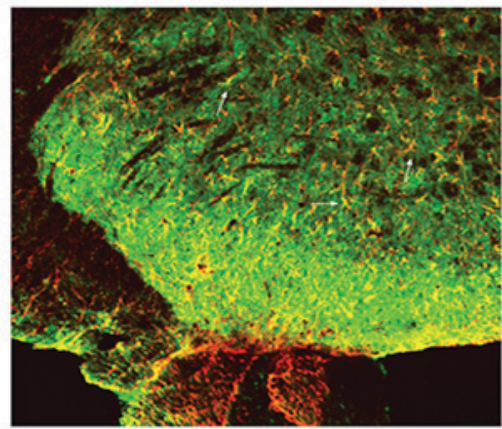

$\alpha 2$ AR/GFAP

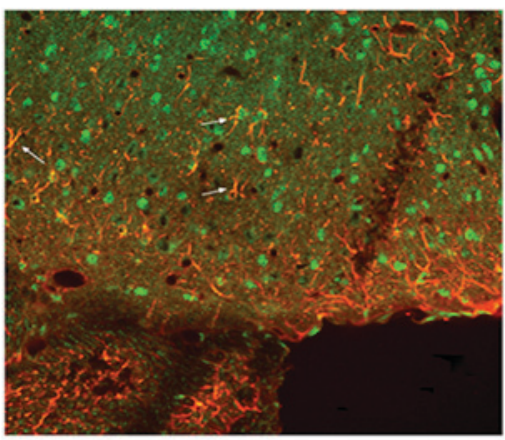

TLR4/GFAP

B
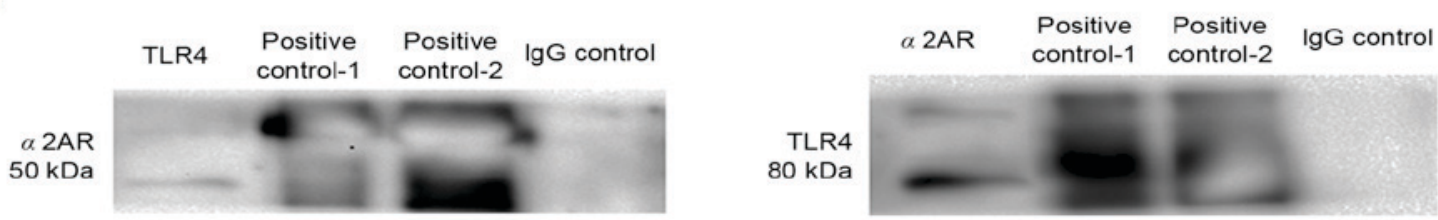

Figure 5. (A) Double immunofluorescence revealed that GFAP was co-localized with $\alpha 2 \mathrm{AR}$ and TLR4 immunoreactivity in the spinal dorsal horn of the CFA group at 3 days. Arrowheads indicate double-labeled cells. (B) Co-immunoprecipitation assay. $\alpha 2 A R$ was detected by with specific antibodies to TLR4, and TLR4 was also detected with specific antibodies to $\alpha 2 \mathrm{AR}$, as was the positive control. However, when IgG was used as a negative control, no immunoreactivity was detected. Magnification, x20. IgG, immunoglobulin G; GFAP, glial fibrillary acid protein; TLR, Toll-like receptor; $\alpha 2$ AR, $\alpha$ 2-adrenoceptors.

between $\alpha 2 \mathrm{AR}$ and TLR 4 was assessed through co-immunoprecipitation. The results indicated that after immunoprecipitation, TLR4 was detected with the specific antibody for $\alpha 2 A R$, and $\alpha 2 \mathrm{AR}$ was also detected with the specific antibody for TLR4, but these proteins were not detected with normal $\mathrm{IgG}$ as a negative control. These results indicated that $\alpha 2 \mathrm{AR}$ may interact with TLR4 in the spinal dorsal horn at a certain level (Fig. 5B).

\section{Discussion}

The present study investigated the anti-nociceptive effects of DEX on inflammatory responses in CFA-induced MA rats. It was demonstrated that intrathecal administration of $2.5 \mu \mathrm{g}$ DEX for 3 consecutive days significantly attenuated mechanical and thermal hyperalgesia, decreased the production of IL-1 $\beta$, IL- 6 and TNF- $\alpha$, and suppressed the activation of the TLR4/NF- $\kappa \mathrm{B}$ p65 pathway in the spinal tissues of rats with CFA-induced MA. All these effects were markedly reversed by pre-treatment with BRL44408, a selective $\alpha 2 \mathrm{AR}$ antagonist.

$\alpha 2 \mathrm{AR}$ is located diffusely in the nervous system, including primary afferent nerves, spinal dorsal horn neurons and the brainstem (31). As a highly specific potent and selective
2 AR agonist, DEX has been widely used for sedation and analgesia in clinical therapy (2). The main site of action of a2AR agonists, including DEX, is the spinal cord $(32,33)$. DEX exerts dose-dependent analgesic effects against inflammation as well as neuropathic and post-operative pain. To further reveal the effect of DEX on neuropathic pain, the present study investigated the anti-nociceptive effects of DEX in rats with CFA-induced MA, and found that repeated intrathecal administration of DEX for 3 consecutive days after CFA injection, starting from the early phase of inflammation, significantly attenuated mechanical and thermal hyperalgesia. Although the present and previous studies have demonstrated the anti-nociceptive effects of DEX on CFA-induced MA rats, the underlying molecular mechanisms still remain largely elusive (2,34).

TLR4, a transmembrane receptor protein, functions as a signaling transduction molecule through its extracellular leucine-rich repeated domains and a cytoplasmic signaling domain. It has been demonstrated that TLR4 not only has a key role in the innate immune response, but also participates in the triggering of adaptive immunity (35-38). While LPS is well recognized as a major ligand of TLR4 through interaction with CD14, endogenous ligands for TLR4 also likely exist. For 
instance, the association of TLR4 with myeloid differentiation factor 88 (MYD88) has been demonstrated to activate the TNF receptor- and IL-1 receptor-associated kinases, which further leads to inflammatory responses $(35,39)$.

$\mathrm{NF}-\kappa \mathrm{B}$, a key nuclear transcription factor, generally consists of the p50 and p65 subunits. It has been well-established that $\mathrm{NF}-\kappa \mathrm{B}$ regulates the expression of pro-inflammatory cytokines, and thus has an essential role in immune and inflammatory responses. Furthermore, abnormal activation of the $\mathrm{NF}-\kappa \mathrm{B}$ signaling has been implicated in the pathogenesis of acute and chronic inflammatory diseases $(40,41)$. In the inactive state, $N F-\kappa B$ is located in the cytosol through binding to $\mathrm{NF}-\kappa \mathrm{B}$ inhibitory protein $(\mathrm{I} \kappa \mathrm{B} \alpha)$. Activation of $N F-\kappa B$ induces the release and degradation of $\mathrm{I} \kappa \mathrm{B} \alpha$ from the dimeric complex, followed by phosphorylation of NF- $\kappa \mathrm{B}$ p65 as well as its translocation into the nucleus (42). Once entering into the nucleus, $\mathrm{NF}-\kappa \mathrm{B}$ initiates the gene transcription of pro-inflammatory cytokines, including IL-1 $\beta$, IL-6 and TNF- $\alpha$ (43).

Furthermore, TLR4 mainly activates the downstream effector NF- $\kappa \mathrm{B}$ through MYD88-dependent or -independent pathways. In the present study, the MA model was established by CFA. The findings indicated that TLR4 expression and NF- $\mathrm{B}$ p 65 activation were significantly upregulated in the spinal cord of MA rats, accompanied with mechanical and thermal hyperalgesia. Furthermore, treatment with DEX significantly decreased the CFA-induced upregulation of TLR4 expression and NF- $\mathrm{B}$ p 65 activation in rat spinal cords, along with attenuated mechanical and thermal hyperalgesia, as well as reduced production of pro-inflammatory IL-1 $\beta$, IL- 6 and TNF- $\alpha$. Since TLR4 is an essential upstream sensor for $\mathrm{NF}-\kappa \mathrm{B}$ p 65 activation, and $\mathrm{NF}-\kappa \mathrm{B}$ p 65 activation increases the production of these inflammatory cytokines, it is possible that DEX reduces the production of these inflammatory cytokines by inhibiting the activation of the TLR4/NF- $\mathrm{B}$ p65 signaling pathway in spinal cord of MA rats induced by CFA. These results suggested that inhibition of TLR4/NF- $\mathrm{B}$ p65 signaling is a probable mechanism through which DEX inhibits the production of inflammatory cytokines and thus exerts its anti-inflammatory effects, which contribute to its anti-nociceptive effects in CFA-induced MA rats.

In conclusion, the present study demonstrated that DEX attenuates mechanical and thermal hyperalgesia in rats with CFA-induced MA, probably through decreasing the production of pro-inflammatory cytokines. The anti-nociceptive effect of DEX may be associated with downregulation of TLR4 and NF- $\kappa \mathrm{B}$ signaling. Furthermore, $\alpha 2 \mathrm{AR}$ interacted with TLR4, which may have compromised the activation of the TLR4 pathway. These properties are probably associated with the anti-nociceptive effects of DEX in the CNS.

\section{References}

1. Schaible HG, Ebersberger A and Von Banchet GS: Mechanisms of pain in arthritis. Ann N Y Acad Sci 966: 343-354, 2002.

2. Xu B, Zhang WS, Yang JL, Xu H, Deng XM and Zhang YQ: Dexmedetomidine blocks thermal hyperalgesia and spinal glial activation in rat model of monoarthritis. Acta Pharmacol Sin 31: 523-530, 2010.

3. Virtanen R, Savola JM, Saano V and Nyman L: Characterization of the selectivity, specificity and potency of medetomidine as an alpha 2-adrenoceptor agonist. Eur J Pharmacol 150: 9-14, 1988.
4. Shimode N, Fukuoka T, Tanimoto M, Tashiro C, Tokunaga A and Noguchi K: The effects of dexmedetomidine and halothane on Fos expression in the spinal dorsal horn using a rat postoperative pain model. Neurosci Lett 343: 45-48, 2003.

5. Malmberg AB, Hedley LR, Jasper JR, Hunter JC and Basbaum AI: Contribution of alpha(2) receptor subtypes to nerve injury-induced pain and its regulation by dexmedetomidine. $\mathrm{Br} \mathrm{J}$ Pharmacol 132: 1827-1836, 2001.

6. Xu M, Kontinen VK and Kalso E: Effects of radolmidine, a novel alpha2-adrenergic agonist compared with dexmedetomidine in different pain models in the rat. Anesthesiology 93: 473-481, 2000.

7. Lee HG, Choi JI, Kim YO and Yoon MH: The role of alpha-2 adrenoceptor subtype in the antiallodynic effect of intraplantar dexmedetomidine in a rat spinal nerve ligation model. Neurosci Lett 557 Pt B: 118-122, 2013.

8. Paqueron X, Conklin D and Eisenach JC: Plasticity in action of intrathecal clonidine to mechanical but not thermal nociception after peripheral nerve injury. Anesthesiology 99: 199-204, 2003.

9. Pertovaara A and Wei H: Attenuation of ascending nociceptive signals to the rostroventromedial medulla induced by a novel alpha2-adrenoceptor agonist, MPV-2426, following intrathecal application in neuropathic rats. Anesthesiology 92: 1082-1092, 2000.

10. O'Neill L: The Toll/interleukin-1 receptor domain: A molecular switch for inflammation and host defence. Biochem Soc Trans 28: 557-563, 2000.

11. Akira S and Takeda K: Toll-like receptor signalling. Nat Rev Immunol 4: 499-511, 2004.

12. Baeuerle PA and Henkel T: Function and activation of NF-kappa B in the immune system. Annu Rev Immunol 12: 141-179, 1994.

13. Watkins LR, Milligan ED and Maier SF: Glial activation: A driving force for pathological pain. Trends Neurosci 24: 450-455, 2001.

14. Tsuda M, Inoue K and Salter MW: Neuropathic pain and spinal microglia: A big problem from molecules in 'small' glia. Trends Neurosci 28: 101-107, 2005.

15. Cao $\mathrm{H}$ and Zhang YQ: Spinal glial activation contributes to pathological pain states. Neurosci Biobehav Rev 32: 972-983, 2008.

16. Gwak YS, Kang J, Unabia GC and Hulsebosch CE: Spatial and temporal activation of spinal glial cells: Role of gliopathy in central neuropathic pain following spinal cord injury in rats. Exp Neurol 234: 362-372, 2012.

17. Milligan ED, Twining C, Chacur M, Biedenkapp J, O'Connor K, Poole S, Tracey K, Martin D, Maier SF and Watkins LR: Spinal glia and proinflammatory cytokines mediate mirror-image neuropathic pain in rats. J Neurosci 23: 1026-1040, 2003.

18. Ledeboer A, Sloane EM, Milligan ED, Frank MG, Mahony JH, Maier SF and Watkins LR: Minocycline attenuates mechanical allodynia and proinflammatory cytokine expression in rat models of pain facilitation. Pain 115: 71-83, 2005.

19. Sun S, Cao H, Han M, Li TT, Pan HL, Zhao ZQ and Zhang YQ: New evidence for the involvement of spinal fractalkine receptor in pain facilitation and spinal glial activation in rat model of monoarthritis. Pain 129: 64-75, 2007.

20. Shan S, Qi-Liang MY, Hong C, Tingting L, Mei H, Haili P, Yan-Qing W, Zhi-Qi Z and Yu-Qiu Z: Is functional state of spinal microglia involved in the anti-allodynic and anti-hyperalgesic effects of electroacupuncture in rat model of monoarthritis? Neurobiol Dis 26: 558-568, 2007.

21. Sun S, Cao H, Han M, Li TT, Zhao ZQ and Zhang YQ: Evidence for suppression of electroacupuncture on spinal glial activation and behavioral hypersensitivity in a rat model of monoarthritis. Brain Res Bull 75: 83-93, 2008.

22. Sun S, Chen WL, Wang PF, Zhao ZQ and Zhang YQ: Disruption of glial function enhances electroacupuncture analgesia in arthritic rats. Exp Neurol 198: 294-302, 2006.

23. Mori K, Ozaki E, Zhang B, Yang L, Yokoyama A, Takeda I, Maeda N, Sakanaka M and Tanaka J: Effects of norepinephrine on rat cultured microglial cells that express alpha1, alpha2, beta 1 and beta 2 adrenergic receptors. Neuropharmacology 43: 1026-1034, 2002.

24. Peng L, Yu AC, Fung KY, Prévot V and Hertz L: Alpha-adrenergic stimulation of ERK phosphorylation in astrocytes is alpha(2)-specific and may be mediated by transactivation. Brain Res 978: 65-71, 2003.

25. Heal DJ, Butler SA, Prow MR and Buckett WR: Quantification of presynaptic alpha 2-adrenoceptors in rat brain after short-term DSP-4 lesioning. Eur J Pharmacol 249: 37-41, 1993. 
26. Conway EL, Gundlach AL and Craven JA: Temporal changes in glial fibrillary acidic protein messenger RNA and [3H]PK11195 binding in relation to imidazoline-I2-receptor and alpha 2-adrenoceptor binding in the hippocampus following transient global forebrain ischaemia in the rat. Neuroscience 82: 805-817, 1998.

27. Li SS, Zhang WS, Ji D, Zhou YL, Li H, Yang JL, Xiong YC, Zhang YQ and Xu H: Involvement of spinal microglia and interleukin-18 in the anti-nociceptive effect of dexmedetomidine in rats subjected to CCI. Neurosci Lett 560: 21-25, 2014.

28. Xu JJ, Walla BC, Diaz MF, Fuller GN and Gutstein HB: Intermittent lumbar puncture in rats: A novel method for the experimental study of opioid tolerance. Anesth Analg 103: 714-720, 2006.

29. Chu YX, Zhang YQ and Zhao ZQ: Involvement of microglia and interleukin-18 in the induction of long-term potentiation of spinal nociceptive responses induced by tetanic sciatic stimulation. Neurosci Bull 28: 49-60, 2012.

30. Yang JL, Xu B, Li SS, Zhang WS, Xu H, Deng XM and Zhang YQ: Gabapentin reduces CX3CL1 signaling and blocks spinal microglial activation in monoarthritic rats. Mol Brain 5 $18,2012$.

31. Unnerstall JR, Kopajtic TA and Kuhar MJ: Distribution of alpha 2 agonist binding sites in the rat and human central nervous system: Analysis of some functional, anatomic correlates of the pharmacologic effects of clonidine and related adrenergic agents. Brain Res 319: 69-101, 1984

32. Liu L, Ji F, Liang J, He H, Fu Y and Cao M: Inhibition by dexmedetomidine of the activation of spinal dorsal horn glias and the intracellular ERK signaling pathway induced by nerve injury. Brain Res 1427: 1-9, 2012.

33. Buerkle $\mathrm{H}$ and Yaksh TL: Pharmacological evidence for different alpha 2-adrenergic receptor sites mediating analgesia and sedation in the rat. Br J Anaesth 81: 208-215, 1998.

34. Xu B, Zhang WS, Yang JL, Lû N, Deng XM, Xu H and Zhang YQ: Evidence for suppression of spinal glial activation by dexmedetomidine in a rat model of monoarthritis. Clin Exp Pharmacol Physiol 37: e158-e166, 2010.
35. Wu Y, Liu Y, Huang H, Zhu Y, Zhang Y, Lu F, Zhou C, Huang L, $\mathrm{Li} X$ and Zhou C: Dexmedetomidine inhibits inflammatory reaction in lung tissues of septic rats by suppressing TLR4/NF- $\mathrm{B}$ pathway. Mediators Inflamm 2013: 562154, 2013.

36. Bettoni I, Comelli F, Rossini C, Granucci F, Giagnoni G, Peri F and Costa B: Glial TLR4 receptor as new target to treat neuropathic pain: Efficacy of a new receptor antagonist in a model of peripheral nerve injury in mice. Glia 56: 1312-1319, 2008

37. Saito O, Svensson CI, Buczynski MW, Wegner K, Hua XY, Codeluppi S, Schaloske RH, Deems RA, Dennis EA and Yaksh TL: Spinal glial TLR4-mediated nociception and production of prostaglandin E(2) and TNF. Br J Pharmacol 160: 1754-1764, 2010.

38. Wu GJ, Chen TL, Ueng YF and Chen RM: Ketamine inhibits tumor necrosis factor-alpha and interleukin-6 gene expressions in lipopolysaccharide-stimulated macrophages through suppression of toll-like receptor 4-mediated c-Jun N-terminal kinase phosphorylation and activator protein-1 activation. Toxicol Appl Pharmacol 228: 105-113, 2008

39. Feng Y and Longmore GD: The LIM protein Ajuba influences interleukin-1-induced NF-kappaB activation by affecting the assembly and activity of the protein kinase Czeta/p62/TRAF6 signaling complex. Mol Cell Biol 25: 4010-4022, 2005.

40. Wright JG and Christman JW: The role of nuclear factor kappa $\mathrm{B}$ in the pathogenesis of pulmonary diseases: Implications for therapy. Am J Respir Med 2: 211-219, 2003.

41. Wu Y, Li W, Zhou C, Lu F, Gao T, Liu Y, Cao J, Zhang Y, Zhang Y and Zhou C: Ketamine inhibits lipopolysaccharide-induced astrocytes activation by suppressing TLR4/NF- $\kappa \mathrm{B}$ pathway. Cell Physiol Biochem 30: 609-617, 2012.

42. Baeuerle PA and Baltimore D: I kappa B: A specific inhibitor of the NF-kappa B transcription factor. Science 242: 540-546, 1988.

43. Grilli M, Chiu JJ and Lenardo MJ: NF-kappa B and Rel: Participants in a multiform transcriptional regulatory system. Int Rev Cytol 143: 1-62, 1993. 